\title{
A NOTE ON QUASI-TOPOLOGICAL SPACES
}

\author{
Won Keun Min
}

\begin{abstract}
We study some properties of quasi-topological spaces Also we introduce the concept of $Q$-continuity and investigate several types of quasi-topology on an SGNS.
\end{abstract}

\section{Introduction and preliminaries}

In $[2,3]$, Császár introduced the notion of quasi-topology which is a generalized topology [1]. In [6], the author introduced the strong generalized neighborhood systems which are generalizations of neighborhood systems. The strong generalized neighborhood system induces a strong generalized neighborhood space (briefly SGNS). We introduced the two concepts of interior and closure on an SGNS and investigate some properties in $[6,7]$. In this paper, we introduce and study the notions of $Q$-continuity in a $q$-space. In particular, we investigate several types of quasi-topology on an SGNS.

Definition $1.1([6])$. Let $X$ be a nonempty set with the power set $\exp (X)$ and $\psi: X \rightarrow \exp (\exp (X))$. Then $\psi$ is called a strong generalized neighborhood system on $X$ if it satisfies the following:

(1) $x \in V$ for $V \in \psi(x)$;

(2) for $U, V \in \psi(x), V \cap U \in \psi(x)$.

Then the pair $(X, \psi)$ is called a strong generalized neighborhood space (briefly SGNS) on $X$. Then $V \in \psi(x)$ is called a strong generalized neighborhood of $x \in X$.

$\psi: X \rightarrow \exp (\exp (X))$ is called a generalized neighborhood system [1] on $X$ if it satisfies $x \in V$ for $V \in \psi(x)$. It is obvious that every strong generalized neighborhood system is a generalized neighborhood system.

Received July 23, 2010. Accepted February 8, 2011.

2000 Mathematics Subject Classification. 54A10, 54C08

Key words and phrases. strong generalized neighborhood systems, SGNS, $q$-open, $q$-closed, quasi-topology, $Q$-continuous, $Q$-open map 
Definition $1.2([6])$. Let $(X, \psi)$ be an SGNS on $X$ and $A \subseteq X$. Then the interior and closure of $A$ on $\psi$ (denoted by $\iota(A), \gamma(A)$, respectively) are defined as following:

$\iota(A)=\{x \in A$ : there exists $V \in \psi(x)$ such that $V \subseteq A\} ;$

$\gamma(A)=\{x \in X: V \cap A \neq \emptyset$ for all $V \in \psi(x)\}$.

Theorem $1.3([6])$. Let $(X, \psi)$ be an SGNS on $X$. We obtain the following.

(1) $\iota(A) \subseteq A$ and $A \subseteq \gamma(A)$, for all $A \subseteq X$;

(2) $\iota(A \cap B)=\iota(A) \cap \iota(B)$ and $\gamma(A \cup B)=\gamma(A) \cup \gamma(B)$, for all $A, B \in 2^{X}$

(3) $\gamma(A)=X-\iota(X-A), \iota(A)=X-\gamma(X-A)$.

Definition 1.4 ([7]). Let $(X, \psi)$ be an SGNS on $X$ and $A \subseteq X$. Then the weak- interior and weak-closure of $A$ on $X$ (denoted by $I(A)$, $C(A)$, respectively) are defined as following:

(1) $I(A)=\{x \in A: A \in \psi(x)\}$.

(2) $C(A)=\{x \in X: X-A \notin \psi(x)\}$.

Theorem $1.5([7])$. Let $(X, \psi)$ be an $S G N S$ and $A, B \subseteq X$. Then the following hold.

(1) $I(A) \subseteq A$ and $A \subseteq C(A)$.

(2) $I(A) \cap I(B) \subseteq I(A \cap B)$ and $C(A \cup B) \subseteq C(A) \cup C(B)$.

(3) $I(A)=X-C(X-A)$ and $C(A)=X-I(X-A)$.

(4) $I(A) \subseteq \iota(A)$ and $\gamma(A) \subseteq C(A)$.

A collection $\mathbf{H}$ of subsets of $X$ is called an m-family [5] on $X$ if $\cap \mathbf{H} \neq \emptyset$. Let $\psi$ be an SGNS in $X$ and let $\mathbf{H}$ be an $m$-family on $X$. Then we say that an $m$-family $\mathbf{H}$ converges to $x \in X$ if $\mathbf{H}$ is finer than $\psi(x)$ i.e., $\psi(x) \subseteq \mathbf{H}[6]$. Let $(X, \psi)$ be a SGNS on $X$ and $A \subset X$.

\section{Quasi-topological spaces}

Definition 2.1. Let $X$ be a nonempty set. A subfamily $q_{X}$ of the power set $\exp (X)$ is called a quasi-topology [3] on $X$ if it satisfies the following:

(1) $\emptyset \in q_{X}$.

(2) For $U_{1}, U_{2} \in q_{X}, U_{1} \cap U_{2} \in q_{X}$.

(3) If $U_{i} \in q_{X}$ for $i \in J$, then $\cup_{i \in J} U_{i} \in q_{X}$.

Then the pair $\left(X, q_{X}\right)$ is called a $q$-space on $X$. Then $V \in q_{X}$ is called a $q$-open set and the complement of a $q$-open set is a $q$-closed set. 
The collection of all $q$-open sets (resp. $q$-closed sets) in a $q$-space $X$ will be denoted by $Q O(X)$ (resp. $Q C(X))$. We set $Q(x)=\{U \in$ $Q O(X): x \in U\}$.

Remark 2.2. Every topological space is a $q$-space. Clearly the converse is not always true.

Definition 2.3. Let $\left(X, q_{X}\right)$ be a $q$-space. For a subset $A$ of $X$, the $q$-closure of $A$ and the $q$-interior of $A$ are defined as follows:

(1) $q C l(A)=\cap\left\{F: A \subseteq F, X-F \in q_{X}\right\}$.

(2) $q \operatorname{Int}(A)=\cup\left\{U: U \subseteq A, U \in q_{X}\right\}$.

Theorem 2.4. Let $\left(X, q_{X}\right)$ be a $q$-space and $A \subseteq X$.

(1) $x \in q \operatorname{Int}(A)$ if and only if there exists an element $U \in Q(x)$ such that $U \subseteq A$.

(2) $x \in q C l(A)$ if and only if $A \cap V \neq \emptyset$ for all $V \in Q(x)$.

Proof. It is obvious.

Theorem 2.5. Let $\left(X, q_{X}\right)$ be a $q$-space and $A \subseteq X$.

(1) $A$ is $q$-open if and only if $q \operatorname{Int}(A)=A$.

(2) $A$ is $q$-closed if and only if $q C l(A)=A$.

Proof. From Definition 2.3, it is obvious.

Theorem 2.6. Let $\left(X, q_{X}\right)$ be a $q$-space and $A, B \subseteq X$.

(1) If $A \subseteq B$, then $q \operatorname{Int}(A) \subseteq q \operatorname{Int}(B)$ and $q C l(A) \subseteq q C l(B)$.

(2) $q C l(X-A)=X-q \operatorname{Int}(A)$ and $q \operatorname{Int}(X-A)=X-q C l(A)$.

Proof. (1) and (2) are obvious.

Theorem 2.7. Let $\left(X, q_{X}\right)$ be a $q$-space and $A, B \subset X$.

(1) $q \operatorname{Int}(A) \subseteq A$.

(2) $q \operatorname{Int}(A \cap B)=q \operatorname{Int}(A) \cap q \operatorname{Int}(B)$.

(3) $q \operatorname{Int}(q \operatorname{Int}(A))=q \operatorname{Int}(A)$.

Proof. Obvious.

Theorem 2.8. Let $\left(X, q_{X}\right)$ be a $q$-space and $A, B \subset X$.

(1) $A \subseteq q C l(A)$.

(2) $q C l(A \cup B)=q C l(A) \cup q C l(B)$.

(3) $q C l(q C l(A))=q C l(A)$.

Proof. From Theorem 2.6(2), it is obvious.

Remark 2.9. Let $\left(X, q_{X}\right)$ be a $q$-space. Then in general, $q \operatorname{Int}(X)=$ $X$ and $q C l(\emptyset)=\emptyset$ are not true. For the example, consider $X=\{a, b, c\}$ and a quasi-topology $q_{X}=\{\emptyset,\{a\},\{b\},\{a, b\}\}$. Then $q \operatorname{Int}(X)=\{a, b\} \neq$ $X$ and $q C l(\emptyset)=\{c\} \neq \emptyset$. 


\section{Q-continuity and quasi-topologies on an SNGS}

Definition 3.1. Let $f: X \rightarrow Y$ be a function between $q$-spaces $\left(X, q_{X}\right)$ and $\left(Y, q_{Y}\right)$. Then $f$ is said to be $Q$-continuous if for every $A \in Q O(Y), f^{-1}(A)$ is in $Q O(X)$.

Theorem 3.2. Let $f: X \rightarrow Y$ be a function on two $q$-spaces $\left(X, q_{X}\right)$ and $\left(Y, q_{Y}\right)$. Then the following statements are equivalent:

(1) $f$ is $Q$-continuous.

(2) For every $A \in Q C(Y), f^{-1}(A)$ is in $Q C(X)$.

(3) $f(q C l(A)) \subseteq q C l(f(A))$ for $A \subseteq X$.

(4) $q C l\left(f^{-1}(V)\right) \subseteq f^{-1}(q C l(V))$ for $B \subseteq Y$.

(5) $f^{-1}(q \operatorname{Int}(V)) \subseteq q \operatorname{Int}\left(f^{-1}(V)\right)$ for $B \subseteq Y$.

Proof. (1) $\Leftrightarrow(2)$ Obvious.

$(1) \Rightarrow(3)$ Let $x \in q C l(A)$. If $f(x)$ is not in $q C l(f(A))$, then there is a $q$-open subset $V$ containing $f(x)$ such that $V \cap f(A)=\emptyset$. By $Q$ continuity, $f^{-1}(V)$ is $q$-open and since $f^{-1}(V) \cap A=\emptyset, x \notin q C l(A)$; a contradiction.

$(3) \Rightarrow(4)$ Let $A=f^{-1}(B)$ for $B \subseteq Y$; then by $(3), f(q C l(A)) \subseteq$ $q C l(f(A))=q C l\left(f\left(f^{-1}(B)\right)\right) \subseteq q C l(B)$. Thus we have (4).

$(4) \Rightarrow(5)$ By Theorem 2.6, it is obvious.

$(5) \Rightarrow(1)$ Let $V \in Q O(Y)$. By $(5), f^{-1}(V) \subseteq q \operatorname{Int}\left(f^{-1}(V)\right)$. Thus $f^{-1}(V) \in Q O(X)$.

Let $\left(X, q_{X}\right)$ be a $q$-space and $\mathbf{H}$ an $m$-family on $X$. Then we say that an $m$-family $\mathbf{H} q$-converges to $x \in X$ if $\mathbf{H}$ is finer than $Q(x)$ i.e., $Q(x) \subseteq \mathbf{H}$. Let $f: X \rightarrow Y$ be a function; then it is obvious $f(\mathbf{H})=$ $\{f(F): F \in \mathbf{H}\}$ is an $m$-family on $Y$.

Theorem 3.3. Let $f: X \rightarrow Y$ be a bijective function between $q$ spaces $\left(X, q_{X}\right)$ and $\left(Y, q_{Y}\right)$. Then $f$ is $Q$-continuous iff for an $m$-family $\mathbf{H} q$-converging to $x \in X, f(\mathbf{H}) q$-converges to $f(x)$.

Proof. Suppose $f$ is $Q$-continuous and $\mathbf{H}$ is an $m$-family $q$-converging to $x \in X$. By hypothesis and surjectivity, we get $Q(f(x)) \subseteq f(Q(x)) \subseteq$ $f(\mathbf{H})$, so that $f(\mathbf{H}) q$-converges to $f(x)$.

For the converse, let $U \in Q(f(x))$ for $x \in X$. Since $Q(x) q$-converges to $x$, by hypothesis, we get $Q(f(x)) \subseteq f(Q(x))$. From $f$ is injectivity, it follows $f^{-1}(U) \in Q(x)$. 
Definition 3.4. Let $f: X \rightarrow Y$ be a function between $q$-spaces $\left(X, q_{X}\right)$ and $\left(Y, q_{Y}\right)$. Then $f$ is said to be $q$-open if for every $A \in Q O(X)$, $f(A) \in Q O(Y)$.

Theorem 3.5. Let $f:\left(X, q_{X}\right) \rightarrow\left(Y, q_{Y}\right)$ be a function between two $q$-spaces. Then the following are equivalent:

(1) $f$ is $q$-open.

(2) $f(q \operatorname{Int}(A)) \subseteq q \operatorname{Int}(f(A))$ for $A \subseteq X$.

(3) $q \operatorname{Int}\left(f^{-1}(B)\right) \subseteq f^{-1}(q \operatorname{Int}(B))$ for $B \subseteq Y$.

Proof. (1) $\Rightarrow(2)$ Suppose that $f$ is $q$-open and $y \in f(q \operatorname{Int}(A))$. Then there exists $x \in q \operatorname{Int}(A)$ such that $f(x)=y$, and so there is $U \in Q(x)$ such that $U \subseteq A$. Since $f$ is $q$-open, $y \in f(U) \in Q(f(x))$ and $f(U) \subseteq$ $f(A)$, so that we have $y \in q \operatorname{Int}(f(A))$.

$(2) \Rightarrow(1)$ For the converse, let $A \in Q O(X)$; then by hypothesis $f(A)=f(q \operatorname{Int}(A)) \subseteq q \operatorname{Int}(f(A))$. Hence we have $f(A) \in Q O(Y)$.

$(2) \Leftrightarrow(3)$ Obvious.

Lemma 3.6. Let $(X, \psi)$ be a $S G N S$ and $A, B \subseteq X$. If $A \subseteq B$, then $\iota(A) \subseteq \iota(B)$ and $\gamma(A) \subseteq \gamma(B)$.

Proof. Obvious.

Theorem 3.7. Let $(X, \psi)$ be an $S G N S$ and $A \subseteq X$. Then $q_{\iota}=$ $\{A \subseteq X: \iota(A)=A\}$ is a quasi-topology on $X$.

Proof. It is obvious that $\emptyset \in q_{\iota}$. For $U, V \in q_{\iota}$, by Theorem 1.3(2), $U \cap V \subseteq q_{\iota}$. If $U_{i} \in q_{\iota}$ for $i \in \Lambda$, then $\cup U_{i} \subseteq \cup \iota\left(U_{i}\right) \subseteq \iota\left(\cup U_{i}\right)$. Hence $\cup U_{i} \in q_{\iota}$.

Let $X$ be a nonempty set. We recall that a family $\tau \subseteq \exp (X)$ is called a supratopology on $X$ [4] if $X \in \tau$ and $\tau$ is closed under arbitrary union.

Theorem 3.8. Let $(X, \psi)$ be an $S G N S$. Then $S q_{X}(X)=\{A \subseteq$ $X: A \subseteq \gamma(\iota(A))\}$ is a generalized topology on $X$ and it indeed is a supratopology.

Proof. It is obvious that $\emptyset \in S q_{X}$. Suppose that there exists a $q$-closed subset $F \neq X$ such that $\iota(X) \subseteq F \subseteq X$. Then $X-F$ is a nonempty $q$-open set such that $X-F \cap \iota(X) \neq \emptyset$ and so $\iota(X) \subseteq X-F \cup \iota(X)$. It is a contradiction.

For each $i \in \Lambda$, if $U_{i} \in S q_{X}(X)$, then $U_{i} \subseteq \gamma \iota\left(U_{i}\right) \subseteq \gamma \iota\left(\cup U_{i}\right)$. Hence $\cup U_{i} \in S q_{X}(X)$. 
Theorem 3.9. Let $(X, \psi)$ be an $S G N S$. Then $\alpha q(X)=\{A \subseteq X$ : $S \cap A \in S q_{X}(X)$ for all $\left.S \in S q_{X}(X)\right\}$ is a quasi-topology on $X$ and it indeed is a topology.

Proof. It is obvious that $X, \emptyset \in \alpha q(X)$. Let $A, B \in \alpha q(X)$. Then for all $S \in S q_{X}(X)$, since $(S \cap A) \in S q_{X}(X), S \cap(A \cap B)=((S \cap A) \cap B) \in$ $S q_{X}(X)$. Hence $A \cap B \in \alpha q(X)$.

For each $i \in \Lambda$, if $U_{i} \in \alpha q(X)$, then for all $S \in S q_{X}(X)$ since $(S \cap$ $\left.U_{i}\right) \in S q_{X}(X)$, by Theorem 3.8, $S \cap \cup U_{i}=\cup\left(S \cap U_{i}\right) \in \alpha q(X)$. Hence $\cup U_{i} \in \alpha q(X)$.

Theorem 3.10. Let $(X, \psi)$ be an $S G N S$. If $S \in S q_{X}(X)$ and $U \in q_{\iota}$, then $S \cap U \in S q_{X}(X)$.

Proof. From Theorem 2.4, It is obvious.

Theorem $3.11([7])$. Let $(X, \psi)$ be an SGNS, $A \subseteq X$ and let $\mathbf{B}=$ $\{A \subseteq X: I(A)=A\}$. Then $q_{I}=\{\cup \sigma: \sigma \subseteq \mathbf{B}\}$ is a quasi-topology on $X$.

Theorem 3.12. Let $(X, \psi)$ be an SGNS. Then $q_{\iota}$ is finer than $q_{I}$.

Proof. From Definition 1.2 and Definition 1.5, it is obvious.

Remark 3.13. Let $(X, \psi)$ be an $S G N S$. Then the inclusions are satisfied:

$$
q_{I} \subseteq q_{\iota} \subseteq \alpha q(X) \subseteq S q_{X}(X)
$$

\section{References}

1. Á. Császár; Generalized Topology, Generalized Continuity, Acta Math. Hungar., 96 (2002), 351-357.

2. Á. Császár; Remark On Quasi-Topologies, Acta Math. Hungar., 119(1-2) (2008), 197-200.

3. Á. Császár; Further remarks on the formular for $\gamma$-interior, Acta Math. Hungar., 113(2006), 325-332.

4. A. S. Mashhour, A. A. Allam, F. S. Mahmoud and F. H. Khedr; On supratopological spaces, Indian J. pure appl. Math., 14(4) (1983), 502-510.

5 . W. K. Min; Some results on generalized topological spaces and generalized systems, Acta Math. Hungar., 108 (1-2) (2005), 171-181.

6. W. K. Min; On Strong Generalized Neighborhood Systems and sg-open sets, Commun. Korean Math. Soc., 23(1) (2008), 125-131.

7. W. K. Min; Results On Strong Generalized Neighborhood Spaces, J. Korea Soc. Math. Educ. Ser. B: Pure Appl. Math., 15(3)(2008), 221-227. 
Won Keun Min

Department of Mathematics, Kangwon National University,

Chuncheon, 200-701, Korea.

E-mail: wkmin@kangwon.ac.kr 